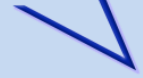

Archive for Organic Chemistry
Arkivoc 2017, part iii, 73-86

\title{
Synthesis and physicochemical properties of merocyanine dyes based on dihydropyridine and fragments of cyanoacetic acid derivatives
}

\author{
Irina A. Borisova, * Andrey A. Zubarev, Lyudmila A. Rodinovskaya, and Anatoliy M. Shestopalov \\ N. D. Zelinsky Institute of Organic Chemistry, Russian Academy of Sciences, \\ 47 Leninsky prosp., 119991 Moscow, Russia \\ E-mail: irdbor@gmail.com
}

Dedicated to Prof. Oleg Rakitin on the occasion of his $65^{\text {th }}$ birthday

Received 10-20-2016

Accepted 12-30-2016

Published on line 03-30-2017

\begin{abstract}
Merocyanine dyes of the dihydropyridine series were prepared from salts of $\alpha(\gamma)$-picolinium and cyanoacetic acid derivatives. Their spectral characteristics suggesting their structure in solution were studied. The change in the spectral properties depending on the substituents introduced into the structure of the substituents and solvents used (solvatochromism) was considered. The protonation of the dyes was studied, and its regioselectivity was established.
\end{abstract}

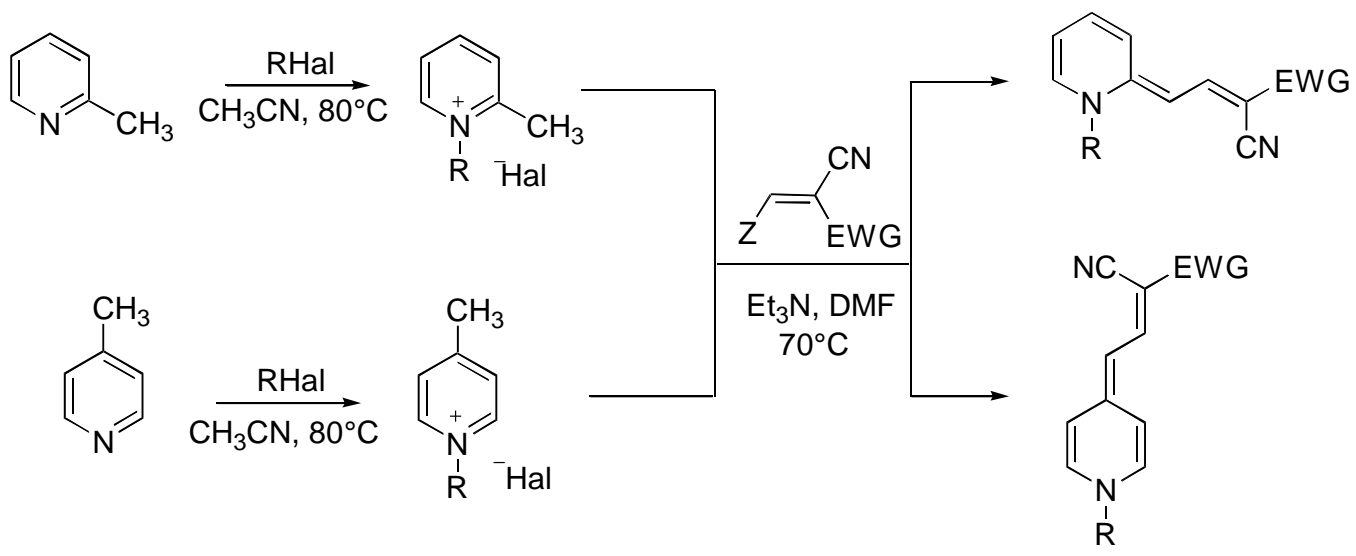

Keywords: Merocyanine dyes; cyanoacetic acid derivatives; picolinium salts; spectral properties; protonation 


\section{Introduction}

Work on the merocyanine dyes, which has intensively been performed since the middle of the 20th century, ${ }^{1-14}$ presently remains urgent. Nowadays interest in these compounds has increased, because these dyes find wide use in many areas of human activity: optoelectronics, photovoltaics, biology, and medicine. 2,15,16 Thermophotoresistors, sensitizers of photographic emulsions, and photochromes are found among the merocyanine dyes. Some such compounds are used as fluorescent markers of cells and various cellular structures. ${ }^{17}$

At the same time, the merocyanine dyes containing the dihydropyridine fragment as a donor moiety and cyanoacetic acid and its derivatives as an acceptor moiety are insufficiently studied, and the data on these compounds are substantially fragmented. ${ }^{18-22}$

This work is devoted to the synthesis of a series of merocyanine dyes based on $\mathrm{N}$-substituted picolinium salts and cyanoacetic acid derivatives, which allows a study of the dependence of the physicochemical properties of the synthesized compounds on the structure.

\section{Results and Discussion}

The target compounds of the 1,2- (6a-I) and 1,4-dihydropyridine structures (7a-i) were synthesized from the corresponding salts of $\alpha$ - or $\gamma$-picolinium salts with different substituents at the nitrogen atoms (Scheme 1 ). Compounds $\mathbf{5 a - c}$ (Table 1 ) were used as the second component. Compounds $\mathbf{5} \mathbf{a}$ and $\mathbf{5} \mathbf{b}$ were synthesized by the condensation of the corresponding derivatives of cyanoacetic acid and triethyl orthoformate. ${ }^{23-24}$ Compound $\mathbf{5 c}$ was obtained by the condensation of cyanothioacetamide, triethyl orthoformate, and aniline, which is more preferable, in this case, than the previous method. ${ }^{25}$ The syntheses of dyes 6 and 7 were carried out in the presence of an excess of base, and dimethylformamide was used as a solvent.

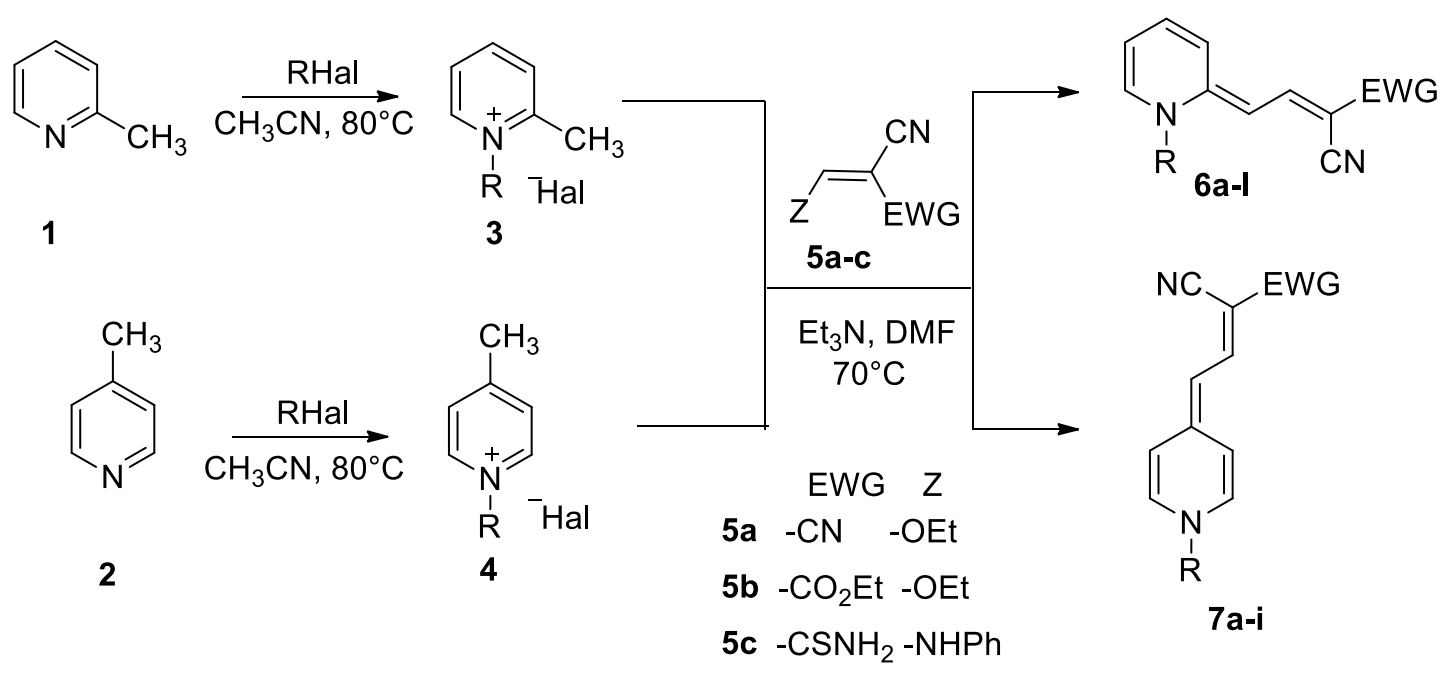

\section{Scheme 1}

The advantages of this method are the simplicity and convenience of target product isolation by precipitation from a DMF solution by dilution with water, an often short time interval of the synthesis, and yields of the target products that are higher than medium values in many cases (Table 1). The reaction is 
highly stereospecific. There is no direct correlation between the properties of substituents and yields of both compounds 6 and 7. Probably there is a complex influence of electronic/steric effects and solubility/polarity properties for isolation and purification procedures. Furthermore the yields for compounds 6 and $\mathbf{7}$ can be related with different stability of betaine form of intermediates (deprotonated picolinium salts).

Table 1. Preparation of compounds 6a-I and 7a-i (Scheme 1)

\begin{tabular}{|c|c|c|c|c|}
\hline Compound & $\mathrm{R}$ & EWG & Time & Yield, \% \\
\hline $6 a$ & $-\mathrm{CH}_{3}$ & $-\mathrm{CN}$ & $5 \mathrm{~min}$ & 78 \\
\hline $6 b$ & $-\mathrm{CH}_{3}$ & $-\mathrm{CO}_{2} \mathrm{Et}$ & $2 \mathrm{~h}$ & 72 \\
\hline $6 c$ & $-\mathrm{CH}_{3}$ & $-\mathrm{CSNH}_{2}$ & $4 \mathrm{~h}$ & 55 \\
\hline $6 d$ & $-B u$ & $-\mathrm{CN}$ & $2 \mathrm{~h}$ & 82 \\
\hline $6 e$ & $-B u$ & $-\mathrm{CO}_{2} \mathrm{Et}$ & $4 \mathrm{~h}$ & 75 \\
\hline $6 f$ & $-B u$ & $-\mathrm{CSNH}_{2}$ & $4 \mathrm{~h}$ & 67 \\
\hline $6 g$ & -Oct & $-\mathrm{CN}$ & $4 \mathrm{~h}$ & 86 \\
\hline $6 h$ & -Oct & $-\mathrm{CO}_{2} \mathrm{Et}$ & $4 \mathrm{~h}$ & 84 \\
\hline $6 i$ & -Oct & $-\mathrm{CSNH}_{2}$ & $4 \mathrm{~h}$ & 88 \\
\hline $6 j$ & $-\mathrm{CH}_{2} \mathrm{CO}_{2} t-\mathrm{Bu}$ & $-\mathrm{CN}$ & $2 \mathrm{~h}$ & 85 \\
\hline $6 k$ & $-\mathrm{CH}_{2} \mathrm{CO}_{2} t-\mathrm{Bu}$ & $-\mathrm{CO}_{2} \mathrm{Et}$ & $4 \mathrm{~h}$ & 63 \\
\hline 61 & $-\mathrm{CH}_{2} \mathrm{CO}_{2} t-\mathrm{Bu}$ & $-\mathrm{CSNH}_{2}$ & $4 \mathrm{~h}$ & 63 \\
\hline $7 a$ & $-\mathrm{CH}_{3}$ & $-\mathrm{CN}$ & $5 \mathrm{~min}$ & 68 \\
\hline $7 b$ & $-\mathrm{CH}_{3}$ & $-\mathrm{CO}_{2} \mathrm{Et}$ & $4 \mathrm{~h}$ & 68 \\
\hline 7c & $-\mathrm{CH}_{3}$ & $-\mathrm{CSNH}_{2}$ & $4 \mathrm{~h}$ & 50 \\
\hline $7 d$ & $-B u$ & $-\mathrm{CN}$ & $2 \mathrm{~h}$ & 67 \\
\hline $7 e$ & $-B u$ & $-\mathrm{CO}_{2} \mathrm{Et}$ & $4 \mathrm{~h}$ & 68 \\
\hline $7 f$ & $-B u$ & $-\mathrm{CSNH}_{2}$ & $4 \mathrm{~h}$ & 62 \\
\hline $7 g$ & -Oct & $-\mathrm{CN}$ & $2 \mathrm{~h}$ & 67 \\
\hline 7h & -Oct & $-\mathrm{CO}_{2} \mathrm{Et}$ & $4 \mathrm{~h}$ & 71 \\
\hline $7 \mathbf{i}$ & -Oct & $-\mathrm{CSNH}_{2}$ & $4 \mathrm{~h}$ & 85 \\
\hline
\end{tabular}

An alternative method for the synthesis of the merocyanine systems containing the dihydropyridine fragment is the three-component reaction of salts $\mathbf{3}, \mathbf{C H}$-acids $\mathbf{8}$, and triethyl orthoformate (Scheme 2). The optimization was carried out for compound $\mathbf{6 a}$. All starting compounds were taken in an equal molar ratio. The maximum yield of the product upon optimization was $38 \%$. This approach is rather promising if further optimization can be achieved.<smiles>Cc1cccc[n+]1C</smiles>

(3a)<smiles>N#CCC#N</smiles>

(8)<smiles>CCOC(OCC)OCC</smiles>

(9)<smiles>CCN(CC)OCCN1C=CC=CC1=CC=C(C#N)C#N</smiles>

(6a)

Scheme 2. Three-component route to merocyanines $\mathbf{6 a}$. 
Compounds $\mathbf{6 a - I}$ and $7 \mathbf{7}-\mathbf{i}$ were obtained as a result of the performed study. Compounds $\mathbf{6 a , b}$ and $\mathbf{7 a , b}$ have been described earlier. ${ }^{10}$ Compounds $6 c-I$ and $7 c-i$ were synthesized by us for the first time.

When studying the spectral characteristics of the synthesized merocyanines, we found the broadening of the signal corresponding to the hydrogen atoms of the heteroaromatic ring in positions 3 and 5 in the ${ }^{1} \mathrm{H}$ NMR spectra of the dyes based on 1,4-dihydropyridine, which is explained by the formed "head-to-tail" complex of molecules of this structure. ${ }^{26}$ In the IR spectra of the compounds containing nitrile groups as an acceptor, the absorption bands of the cyano groups are shifted to a range of $2190-2170 \mathrm{~cm}^{-1}$ compared to the cyano groups of benzylidenemalononitrile, whose absorption bands are arranged near $2220 \mathrm{~cm}^{-1} .27$ The two cyano groups are nonequivalent: two absorption bands are observed at 2190 and $2170 \mathrm{~cm}^{-1}$, indicating a stronger participation in conjugation of one of the groups. For the dyes containing the fragment of ethyl cyanoacetate or cyanothioacetamide as an acceptor, the absorption band of the cyano group also lies at $2190 \mathrm{~cm}^{-1}$, whereas the bands of the ester and thioamide groups are arranged at 1660 and $1250 \mathrm{~cm}^{-1}$, respectively. Thus, the signals of these groups are substantially shifted compared to the reference compounds, ${ }^{28,29}$ which suggests that they contribute mainly to the delocalization of the negative charge in the dye molecule. The same regularities are observed for the compounds based on 1,2-dihydropyridine, except for the fact that the ${ }^{1} \mathrm{H}$ NMR spectra have no peak broadening due to the formation of complexes, which is probably related to the lower symmetry of these molecules.

For further study of the properties of the synthesized merocyanine dyes, we studied their protonation properties. ${ }^{30}$ For example, the solution decolorized upon dissolution of compound 6 a in trifluoroacetic acid, indicating interruption of the $\pi, \pi$-conjugation of the polyene chain (Scheme 3 ). This fact is confirmed by the spectral characteristics of the leuco form (6a.1) presented in Figure 1 . The ${ }^{1} \mathrm{H}$ NMR spectra of the leuco form (6a.1) and the starting merocyanine (6a) are presented in Figure 2. It is seen that the signal corresponding to one proton of the polyene chain with a large spin-spin coupling constant (SSCC) at 5.5 ppm disappeared in the spectrum of the leuco form of merocyanine $6 \mathbf{a}$, and a two-proton signal below 4.0 ppm with a lower SSCC appeared.

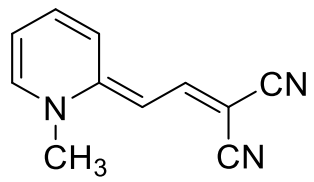

(6a)

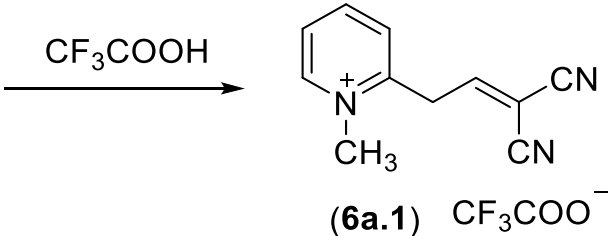

Scheme 3. Protonation of the merocyanine $\mathbf{6 a}$. 


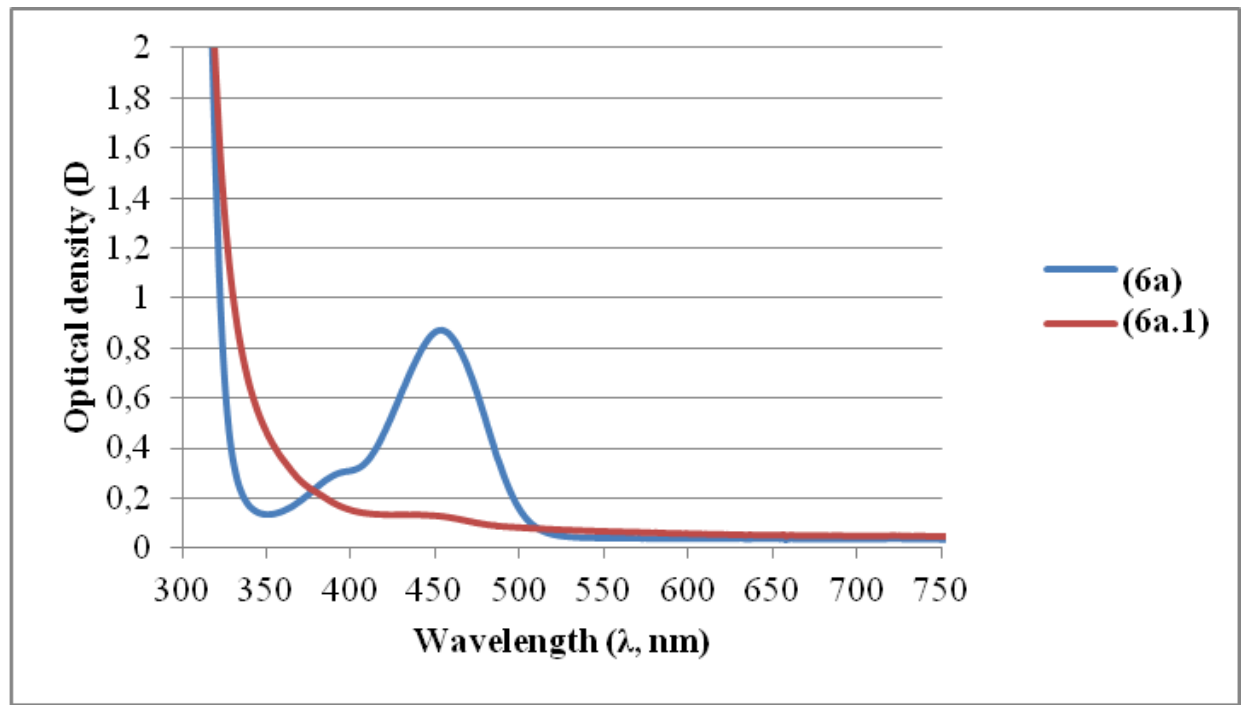

Figure 1. UV/Vis spectra of the merocyanine 6a and its protonated form 6a.1.

(I)

(II)
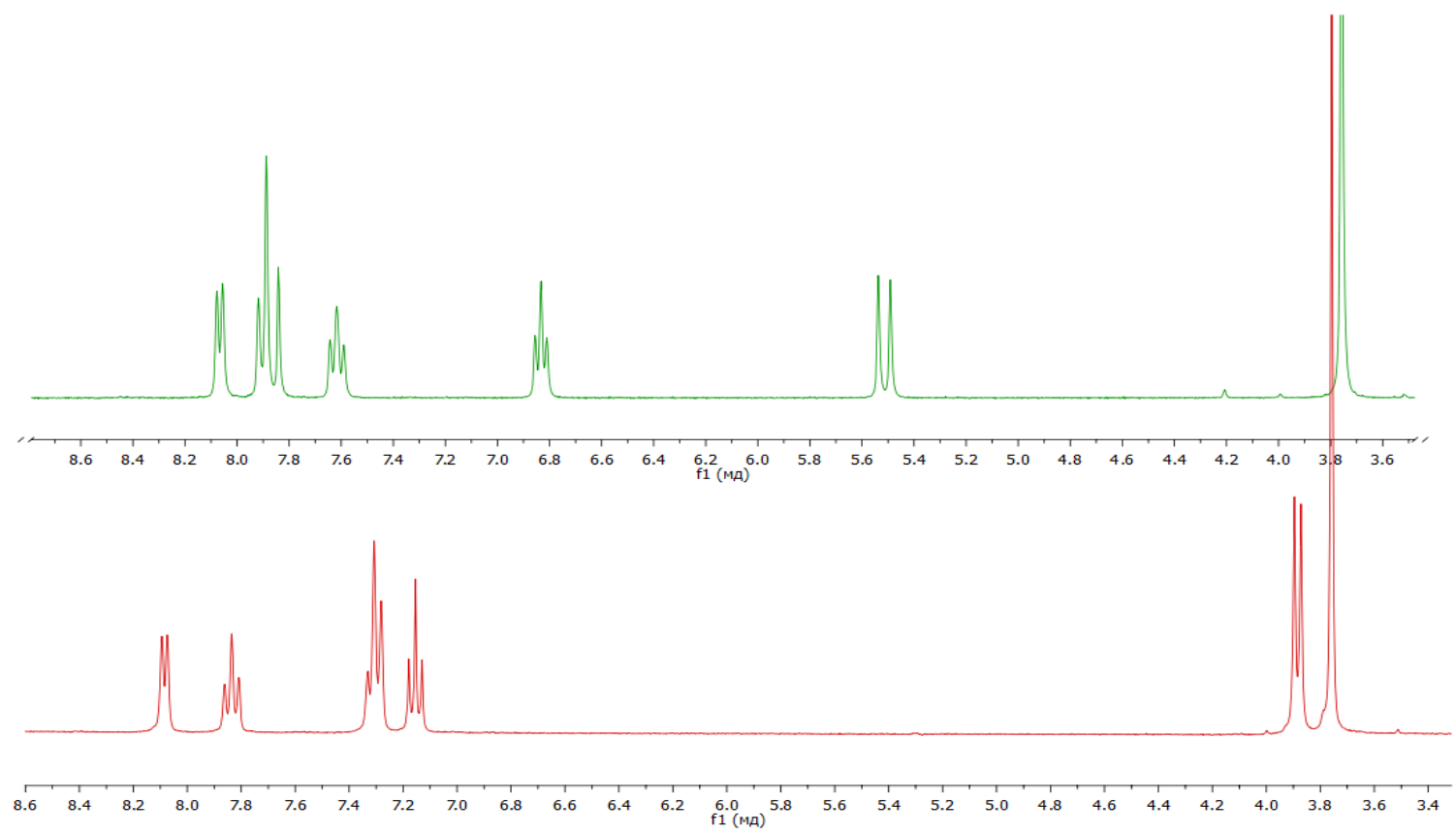

Figure 2. Comparison of the ${ }^{1} \mathrm{H}$ NMR spectra for compounds $6 \mathbf{a}(\mathrm{I})$ and $\mathbf{6 a . 1}$ (II).

According to the 2D COSY experiment, two spin systems were revealed. One of them describes interactions of protons of the heteroaromatic ring, and the second system describes interactions of protons of the lateral chain (Figure 3). The spectral characteristics were detected in a solution of trifluoroacetic acid, because salts of the (6a.1) type were not isolated in the solid state. These compounds are unstable and decompose on an attempt of isolating them in the solid state. Thus, the protonation of the studied dyes occurs highly regioselectively at the $\alpha$-position to the pyridine ring. 


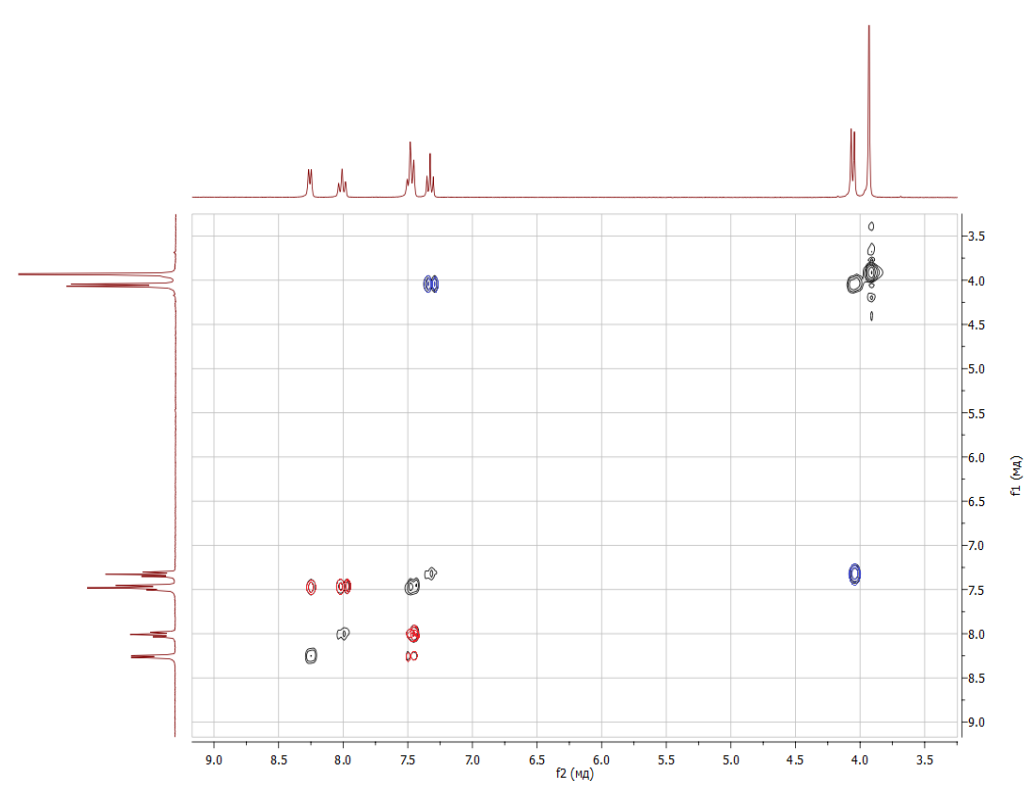

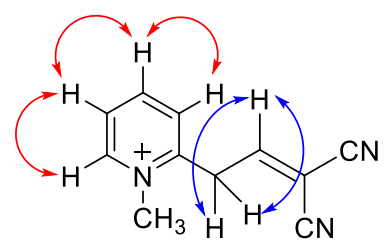

$\mathrm{CF}_{3} \mathrm{COO}$

Figure 3. 2D COSY scan of compound 6a.1 in TFA.

We also measured the UV spectra of the synthesized compounds (Figure 4). For compounds A (6a, 6d, $6 \mathrm{~g}, 6 \mathrm{j}), \mathrm{B}(\mathbf{6 b}, \mathbf{6 e}, \mathbf{6 h}, \mathbf{6 k})$, and $\mathrm{C}(\mathbf{6 c}, 6 \mathrm{f}, 6 \mathrm{i}, 6 \mathrm{l})$, the absorption maximum and molar absorption coefficient increase upon the introduction of new acceptor groups different from the cyano group. For compounds $D$ $(\mathbf{7 a}, \mathbf{7 d}, \mathbf{7 g}), \mathrm{E}(\mathbf{7 b}, \mathbf{7 e}, \mathbf{7 h})$, and $\mathrm{F}(\mathbf{7 c}, \mathbf{7 f}, \mathbf{7 i})$, the absorption maximum also increases but the molar absorption coefficient decreases in the series from the merocyanine dyes based on cyanothioacetamide to the dyes containing cyano group (see Table 2).

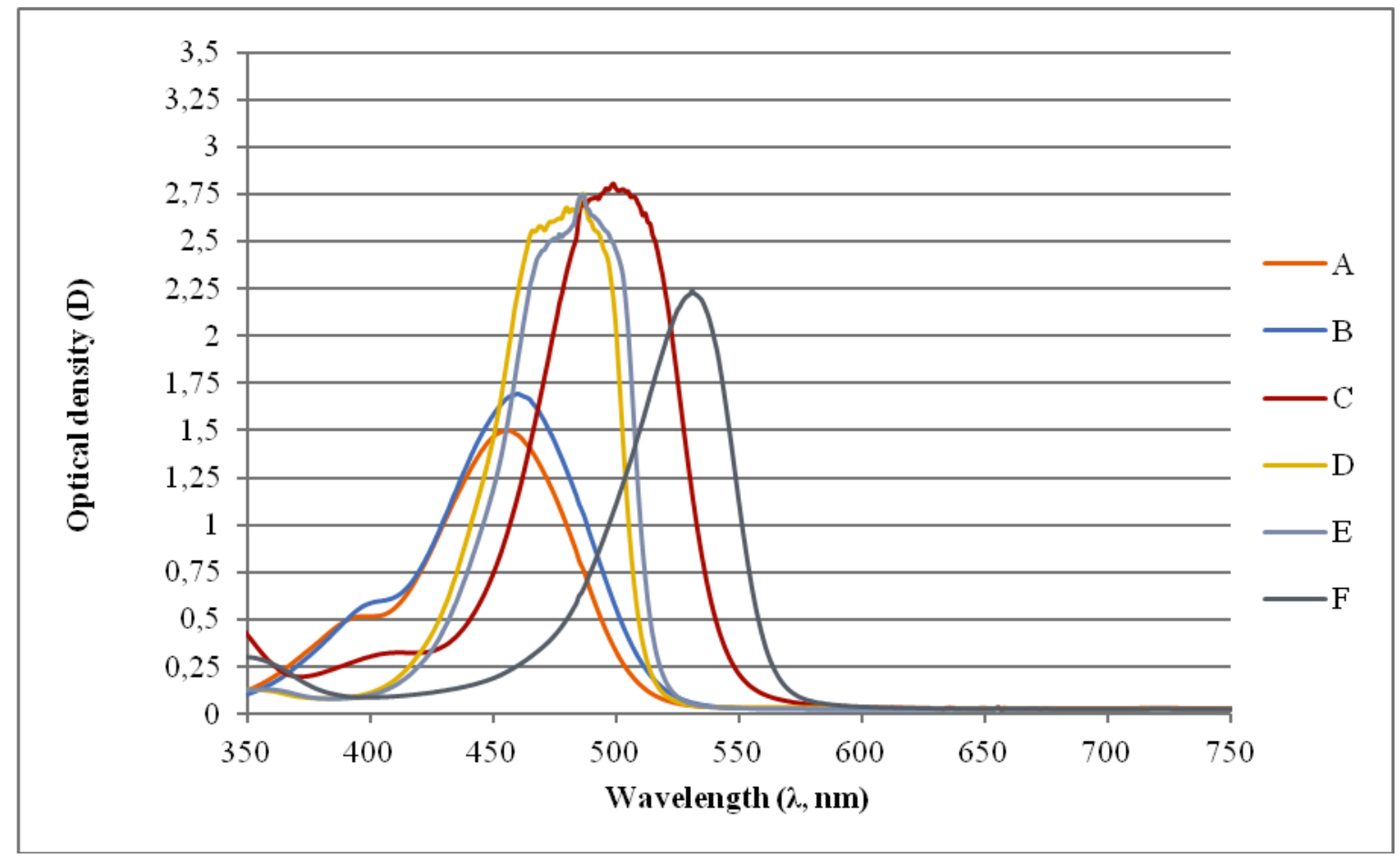

Figure 4. UV/Vis spectra of the merocyanines, grouped according to substituent types. 
Table 2. Absorption spectra of the merocyanine dyes in dichloromethane

\begin{tabular}{cccccc}
\hline Compound & $\lambda_{\max }[\mathrm{nm}]$ & $\begin{array}{c}\varepsilon_{\max } \times 10^{-4} \\
{\left[\mathrm{M}^{-1} . \mathrm{cm}^{-1}\right]}\end{array}$ & Compound & $\lambda_{\max }[\mathrm{nm}]$ & $\begin{array}{c}\varepsilon_{\max } \times 10^{-4} \\
{\left[\mathrm{M}^{-1} \cdot \mathrm{cm}^{-1}\right]}\end{array}$ \\
\hline $\mathbf{6 a}$ & 453 & 3.99 & $\mathbf{6 l}$ & 509 & 4.56 \\
$\mathbf{6 b}$ & 457 & 5.02 & $\mathbf{7 a}$ & 466 & 8.78 \\
$\mathbf{6 c}$ & 502 & 6.61 & $\mathbf{7 b}$ & 486 & 7.71 \\
$\mathbf{6 d}$ & 455 & 4.12 & $\mathbf{7 c}$ & 531 & 4.50 \\
$\mathbf{6 e}$ & 460 & 4.54 & $\mathbf{7 d}$ & 486 & 8.06 \\
$\mathbf{6 f}$ & 496 & 7.14 & $\mathbf{7 e}$ & 486 & 7.61 \\
$\mathbf{6 g}$ & 455 & 4.13 & $\mathbf{7 f}$ & 531 & 5.74 \\
$\mathbf{6 h}$ & 451 & 5.12 & $\mathbf{7 g}$ & 486 & 6.80 \\
$\mathbf{6 i}$ & 469 & 5.42 & $\mathbf{7 h}$ & 486 & 6.40 \\
$\mathbf{6 j}$ & 461 & 3.27 & $\mathbf{7 i}$ & 527 & 6.32 \\
$\mathbf{6 k}$ & 462 & 3.93 & & & \\
\hline
\end{tabular}

Literature data indicate that many merocyanine dyes show pronounced solvatochromism. ${ }^{2}$ Therefore, we studied solvatochromism of the synthesized compounds using substance 7c as an example. The results are presented in Figure 5. The shift of the absorption band maximum is $25-30 \mathrm{~nm}$ in solvents of various polarities, that is less then for well known solvatochromic dye Brooker's merocyanine. ${ }^{31}$.

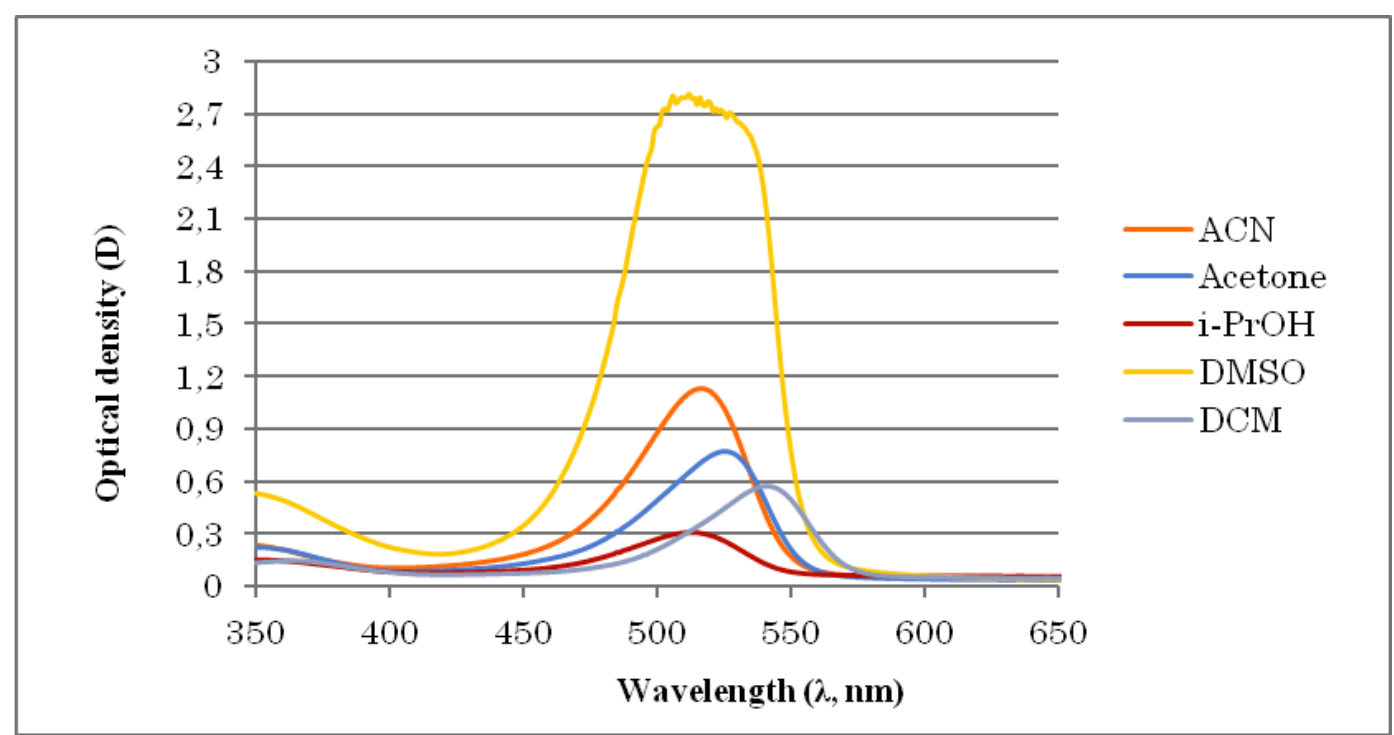

Figure 5. Solvatochromism of compound 7c in solvents of various polarities.

Based on these data and on the fact that the absorption bands are fairly narrow (40-55 $\mathrm{nm}$ at the halfheight of the peaks), we can conclude that the charge delocalization in the studied molecule is high and almost all bonds in the molecule are sesquialteral, i.e., the dye molecule is similar to structure $\mathbf{A} \mathbf{2}$ (Scheme 4), ${ }^{29}$ which is consistent with the published data on these compounds. ${ }^{4}$ 


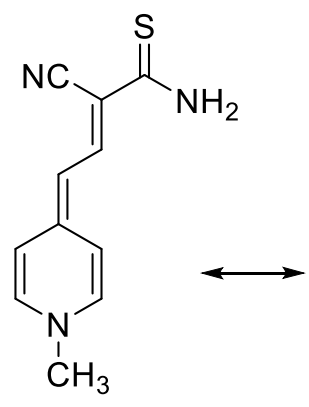

(A1)

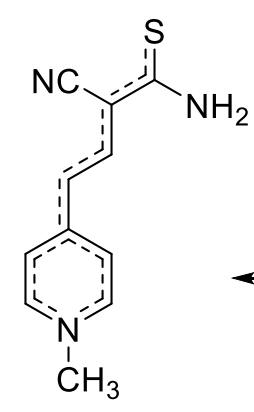

(A2)

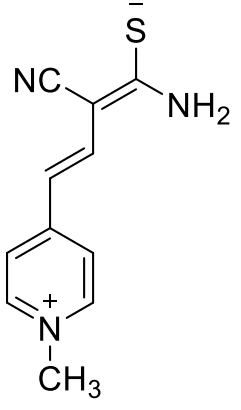

(A3)

Scheme 4. Frontier structures of the merocyanine dyes containing the dihydropyridine fragment.

\section{Conclusions}

(1) A simple, convenient, and stereospecific method is developed for the synthesis of the merocyanine dyes containing the dihydropyridine fragment based on the cyanoacetic acid derivatives.

(2) The spectral characteristics of the merocyanine systems are generalized for the first time. It was found for the compounds containing the 1,2-dihydropyridine fragment that the long-wavelength maximum increased and the molar absorption coefficient decreased with the variation of acceptors at the end of the polyene chain. An opposite regularity is observed for the merocyanines containing the 1,4dihydropyridine fragment.

(3) Solvatochromism observed and previously described in the literature for compounds 7c indicated that described in this work the merocyanine systems similar to the $A 2$ system.

\section{Experimental Section}

General. The structures of the synthesized compounds were confirmed by IR and NMR spectroscopy. IR spectra were recorded on a Bruker ALPHA-T instrument in $\mathrm{KBr}$ pellets. ${ }^{1} \mathrm{H},{ }^{13} \mathrm{C}$, COSY, DEPT, and HMBC NMR spectra were measured on a Bruker AM300 instrument (solvent DMSO- $d_{6}$ ).

UV spectra were recorded on an Agilent 8453 instrument in quartz cells with a light pathlength of $1 \mathrm{~cm}$ with the concentration of the substance $\mathrm{C}_{\mathrm{M}}=10^{-5}[\mathrm{M}]$ (solvent $\mathrm{CH}_{2} \mathrm{Cl}_{2}$ ).

General procedure for synthesis of compounds $5 \mathbf{a}-\mathbf{b}$. A mixture of $\mathrm{CH}$-acid ( 0.1 mol) (malononitrile for $\mathbf{5 a}$ and ethyl cyanoacetate for $\mathbf{5 b})$, triethylorthoformate $(0.1 \mathrm{~mol}, 13 \mathrm{ml})$ and acetic anhydride $(50 \mathrm{ml})$ was stirred at $100{ }^{\circ} \mathrm{C}$ for 4 hours. The resulting reaction mixture was cooled to room temperature and concentrated in vacuo. The solid product was recrystallized from ethanol to afford light yellow crystals. Yields of $\mathbf{5 a} 78 \%\left(\mathrm{mp} 65-67^{\circ} \mathrm{C}\right)^{23}$ and $75 \%$ of $\mathbf{5 b}\left(\mathrm{mp} .49-51^{\circ} \mathrm{C}\right) .^{24}$

Procedure for synthesis of compound 5c. A mixture of cyanothioacetamide $(0.05 \mathrm{~mol}, 5 \mathrm{~g})$, triethyl orthoformate $(0.15 \mathrm{~mol}, 20 \mathrm{ml})$ and aniline $(0.05 \mathrm{~mol}, 4.8 \mathrm{ml})$ was heated with stirring until the exothermic reaction is started. Then the mixture was diluted with ethanol $(20 \mathrm{ml})$, brought to boiling and left to cool down. The dark yellow crystals was filtered and washed with hot ethanol. Yield $78 \%$ (mp. $\left.210-212{ }^{\circ} \mathrm{C}\right) .{ }^{25}$ 
General procedure for synthesis of the merocyanine dyes 6 and 7. A triethylamine excess ( $4 \mathrm{mmol}, 0.48$ $\mathrm{ml}$ ) was added dropwise to a mixture of picolinium salt 3 or 4 (3 mmol) in DMF (1.5 ml), and a $\mathrm{CH}$ acid derivative $5 \mathrm{a}-\mathrm{c}(6 \mathrm{mmol})$ was added. The reaction mixture became colored. Then the reaction mixture was heated at $70^{\circ} \mathrm{C}$ for a period of from $5 \mathrm{~min}$ to $4 \mathrm{~h}$ and then cooled to room temperature. The product was precipitated from the DMF solution by dropwise addition of water. The precipitate formed was filtered off, washed with water, and dried in air. Then the pure merocyanine dyes were isolated by column chromatography on $\mathrm{SiO}_{2}$ using a dichloromethane and acetone (10:1) mixture as an eluent.

(E)-2-(2-(1-Methylpyridin-2(1H)-ylidene)ethylidene)malononitrile (6a). Yield $78 \%, \mathrm{mp}>260{ }^{\circ} \mathrm{C}$, yellow crystals. IR (KBr), v, cm${ }^{-1}$ : 2190, 2170, 1633, 1574, 1536, 1499, 1467, 1451, 1404, 1334, 1275, 1226, 1171, 1058, 1038, 956, 794, 761, 575. ${ }^{1} \mathrm{H}$ NMR, $\delta, \mathrm{ppm}: 3.76\left(\mathrm{~s}, 3 \mathrm{H}, \mathrm{CH}_{3}\right), 5.52\left(\mathrm{~d}, 1 \mathrm{H},(2) \mathrm{CH},{ }^{3} 13.8 \mathrm{~Hz}\right), 6.83(\mathrm{t}, 1 \mathrm{H}$, $\left.\mathrm{CH}_{\mathrm{py}}, J 6.7 \mathrm{~Hz}\right), 7.62\left(\mathrm{t}, 1 \mathrm{H}, \mathrm{CH}_{\mathrm{py}}, J 7.7 \mathrm{~Hz}\right), 7.84-7.92\left(\mathrm{~m}, 2 \mathrm{H},(1) \mathrm{CH}, \mathrm{CH}_{\mathrm{py}}\right), 8.07\left(\mathrm{~d}, 1 \mathrm{H}, \mathrm{CH}_{\mathrm{py}}, J 6.5 \mathrm{~Hz}\right) .{ }^{13} \mathrm{C}$ NMR, $\delta$, ppm: 43.85, 49.35, 93.65, 115.13, 118.87, 120.62, 121.31, 138.52, 142.56, 149.43, 153.69. Anal. calcd for $\mathrm{C}_{11} \mathrm{H}_{9} \mathrm{~N}_{3}$ (183.21): C, 72.11; H, 4.95; N, 22.94. Found: C, 71.95; H, 4.87; N, 22.76 .

Ethyl (2E,4E)-2-cyano-4-(1-methylpyridin-2(1H)-ylidene)but-2-enoate (6b). Yield 72\%, mp $155-157{ }^{\circ} \mathrm{C}$, orange crystals. IR (KBr), v, cm $\mathrm{cm}^{-1}$ : 2192, 1660, 1568, 1536, 1464, 1412, 1344, 1248, 1164, 1100, 1064, 956,

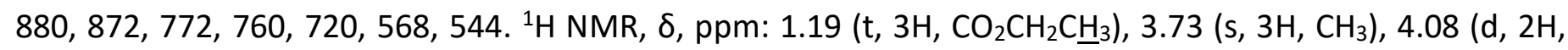
$\left.\mathrm{CO}_{2} \mathrm{C}_{2} \mathrm{CH}_{3}, J 6.4 \mathrm{~Hz}\right), 5.50(\mathrm{~d},(4) \mathrm{CH}, 3 \mathrm{~J} 13.5 \mathrm{~Hz}), 6.77\left(\mathrm{~s}\right.$ br, $\left.1 \mathrm{H}, \mathrm{CH}_{\mathrm{py}}\right), 7.58$ (br.s, $\left.1 \mathrm{H}, \mathrm{CH}_{\mathrm{py}}\right), 7.72\left(\mathrm{~d}, 1 \mathrm{H}, \mathrm{CH}_{\mathrm{py}}, J\right.$ 6.4 Hz), 8.03-8.09 (m, 2H, $\left.\mathrm{CH}_{\mathrm{py}},(3) \mathrm{CH}\right) .{ }^{13} \mathrm{C} \mathrm{NMR,} \delta$, ppm: 15.08, 43.68, 59.49, 75.72, 92.77, 114.41, 120.17, $120.58,138.30,142.54,147.30,154.12,166.82$. Anal. calcd for $\mathrm{C}_{13} \mathrm{H}_{14} \mathrm{~N}_{2} \mathrm{O}_{2}$ (230.27): C, 67.81; $\mathrm{H}, 6.13 ; \mathrm{N}$, 12.17. Found: C, 67.72; H, 5.97; N, 11.97 .

(2E,4E)-2-Cyano-4-(1-methylpyridin-2(1H)-ylidene)but-2-enethioamide (6c). Yield 55\%, mp $200-202{ }^{\circ} \mathrm{C}$, wine-red crystals. IR (KBr), v, cm${ }^{-1}$ : 3396, 3351, 3261, 3150, 2168, 1632, 1620, 1569, 1543, 1458, 1404,

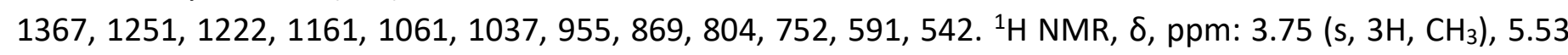
(d, $1 \mathrm{H},(4) \mathrm{CH}, 3$ J $13.7 \mathrm{~Hz}), 6.80\left(\mathrm{t}, 1 \mathrm{H}, \mathrm{CH}_{\mathrm{py}}, J 6.5 \mathrm{~Hz}\right), 7.64\left(\mathrm{t}, 1 \mathrm{H}, \mathrm{CH}_{\mathrm{py}}, J 7.2 \mathrm{~Hz}\right), 7.81\left(\mathrm{~d}, 1 \mathrm{H}, \mathrm{CH}_{\mathrm{py}}, J 8.9 \mathrm{~Hz}\right)$, 8.05-8.07 (m, 3H, NH $\left.2, \mathrm{CH}_{p y}\right), 8.39\left(\mathrm{~d}, 1 \mathrm{H},(3) \mathrm{CH},{ }^{3} \mathrm{~J} 13.7 \mathrm{~Hz}\right) .{ }^{13} \mathrm{C} \mathrm{NMR}, \delta, \mathrm{ppm}: 43.74,89.43,94.08,114.51$, $120.47,129.96,138.15,142.78,144.96,154.27,190.91$. Anal. calcd for $\mathrm{C}_{11} \mathrm{H}_{11} \mathrm{~N}_{3} \mathrm{~S}$ (217.29): C, 60.80; $\mathrm{H}, 5.10$; N, 19.34; S, 14.75. Found: C, 60.72; H, 4.97; N, 19.29; S, 14.68.

(E)-2-(2-(1-Butylpyridin-2(1H)-ylidene)ethylidene)malononitrile (6d). Yield $82 \%, \mathrm{mp} 149-151{ }^{\circ} \mathrm{C}$, orange crystals. IR (KBr), v, cm${ }^{-1}$ : 2956, 2928, 2196, 2172, 1632, 1568, 1536, 1472, 1444, 1404, 1312, 1288, 1224,

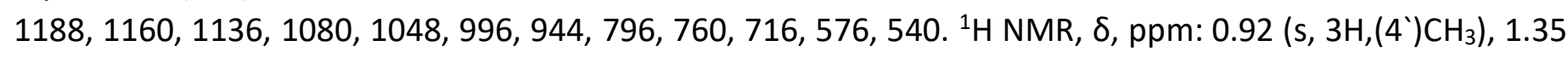
$\left(\mathrm{s}, 2 \mathrm{H},\left(3^{\circ}\right) \mathrm{CH}_{2}\right), 1.69\left(\mathrm{~s}, 2 \mathrm{H},\left(2^{\prime}\right) \mathrm{CH}_{2}\right), 4.12\left(\mathrm{~s}, 2 \mathrm{H},\left(1^{\circ}\right) \mathrm{CH}_{2}\right), 5.57\left(\mathrm{~d}, 1 \mathrm{H},(2) \mathrm{CH}^{3}{ }^{3} \mathrm{~J} 13.3 \mathrm{~Hz}\right), 6.88\left(\mathrm{t}, 1 \mathrm{H}, \mathrm{CH}_{\mathrm{py}}\right)$, $7.64\left(\mathrm{~m}, 1 \mathrm{H}, \mathrm{CH}_{p y}\right), 7.84-7.88\left(\mathrm{~m}, 2 \mathrm{H}, \mathrm{CH}_{\mathrm{py}},(1) \mathrm{CH}\right), 8.05\left(\mathrm{~s}, 1 \mathrm{H}, \mathrm{CH}_{p y}\right) .{ }^{13} \mathrm{C} \mathrm{NMR}, \delta, \mathrm{ppm}: 13.72,19.41,29.81$, 48.56, 55.32, 93.72, 115.74, 119.14, 121.19, 138.82, 142.04, 149.28, 152.68. Anal. calcd for $\mathrm{C}_{14} \mathrm{H}_{15} \mathrm{~N}_{3}$ (225.30): C, 74.64; H, 6.71; N, 18.65. Found: C, 74.59; H, 6.66; N, 18.42.

Ethyl (2E,4E)-2-cyano-4-(1-butylpyridin-2(1H)-ylidene)but-2-enoate (6e). Yield 75\%, mp 91-93 ${ }^{\circ} \mathrm{C}$, orange crystals. IR (KBr), v, cm${ }^{-1}$ : 2967, 2925, 2904, 2875, 2188, 1667, 1634, 1530, 1448, 1418, 1366, 1316, 1290, $1218,1159,1133,1102,1075,1025,958,888,805,753,713,580,538,517 .{ }^{1} \mathrm{H} N M R, \delta, p p m: 0.96(t, 3 \mathrm{H}$, (4 $\left.\left.{ }^{\prime}\right) \mathrm{CH}_{3}, \mathrm{~J} 7.3 \mathrm{~Hz}\right), 1.16-1.23\left(\mathrm{~m}, 7 \mathrm{H}, \mathrm{CO}_{2} \mathrm{CH}_{2} \mathrm{CH}_{3},\left(2^{\prime}, 3^{\prime}\right) \mathrm{CH}_{2}\right), 4.03-4.12\left(\mathrm{~m}, 4 \mathrm{H},\left(1^{\prime}\right) \mathrm{CH}_{2}, \mathrm{CO}_{2} \mathrm{CH}_{2} \mathrm{CH}_{3}\right), 5.55(\mathrm{~d}$, $\left.1 \mathrm{H},(4) \mathrm{CH},{ }^{3} \mathrm{~J} 14.0 \mathrm{~Hz}\right), 6.80\left(\mathrm{t}, 1 \mathrm{H}, \mathrm{CH}_{\mathrm{py}}, \mathrm{J} 6.6 \mathrm{~Hz}\right), 8.01-8.11\left(\mathrm{~m}, 3 \mathrm{H}, 2 \mathrm{CH}_{\mathrm{py}},(3) \mathrm{CH}\right) .{ }^{13} \mathrm{C} \mathrm{NMR}, \delta, \mathrm{ppm}: 13.79$, 15.01, 19.50, 29.66, 54.88, 59.59, 73.21, 92.73, 114.73, 120.88, 138.24, 142.01, 147.41, 153.15, 166.37, 167.04. Anal. calcd for $\mathrm{C}_{16} \mathrm{H}_{20} \mathrm{~N}_{2} \mathrm{O}_{2}$ (272.35): C, 70.56; $\mathrm{H}, 7.40 ; \mathrm{N}, 10.29$. Found: $\mathrm{C}, 70.51 ; \mathrm{H}, 7.18 ; \mathrm{N}, 10.16$.

(2E,4E)-4-(1-Butylpyridin-2(1H)-ylidene)-2-cyanobut-2-enethioamide (6f). Yield 67\%, mp $185-186{ }^{\circ} \mathrm{C}$, winered crystals. IR (KBr), v, cm${ }^{-1}$ : 3346, 3291, 3189, 2956, 2926, 2861, 2177, 1629, 1541, 1412, 1366, 1306, 
$1221,1151,1077,1038,964,869,809,757,720,650,600,559,505 .{ }^{1} \mathrm{H} \mathrm{NMR}, \delta, \mathrm{ppm}: 0.94\left(\mathrm{t}, 3 \mathrm{H},\left(4^{\prime}\right) \mathrm{CH}_{3} \mathrm{~J}^{\mathrm{J}}\right.$ $7 \mathrm{~Hz}), 1.32-1.40\left(\mathrm{~m}, 2 \mathrm{H},\left(3^{\prime}\right) \mathrm{CH}_{2}\right), 1.69-1.74\left(\mathrm{~m}, 2 \mathrm{H},\left(2^{\prime}\right) \mathrm{CH}_{2}\right), 4.13\left(\mathrm{t}, 2 \mathrm{H},\left(1^{\prime}\right) \mathrm{CH}_{2}, J \mathrm{~Hz}\right), 5.57\left(\mathrm{~d}, 1 \mathrm{H},(4) \mathrm{CH}, 3^{3}\right.$ 13.7 Hz), $6.82\left(\mathrm{t}, 1 \mathrm{H}, \mathrm{CH}_{\mathrm{py}}, J 6.3 \mathrm{~Hz}\right), 7.63\left(\mathrm{t}, 1 \mathrm{H}, \mathrm{CH}_{\mathrm{py}}, J 7.3 \mathrm{~Hz}\right), 7.82\left(\mathrm{~d}, 1 \mathrm{H}, \mathrm{CH}_{\mathrm{py}}, J 9 \mathrm{~Hz}\right), 7.98-8.05(\mathrm{~m}, 3 \mathrm{H}$, $\left.\mathrm{NH}_{2}, \mathrm{CH}_{\text {py }}\right), 8.38$ (d, $\left.1 \mathrm{H},(3) \mathrm{CH},{ }^{3} \mathrm{~J} 13.7 \mathrm{~Hz}\right) .{ }^{13} \mathrm{C} \mathrm{NMR}, \delta, \mathrm{ppm}: 13.82,19.53,29.70,55.17,89.36,94.17,114.84$, $120.54,121.06,138.20,142.25,144.80,159.27,190.75$. Anal. calcd for $\mathrm{C}_{14} \mathrm{H}_{17} \mathrm{~N}_{3} \mathrm{~S}$ (259.37): C, 64.83; $\mathrm{H}, 6.61$; N, 16.20; S, 12.36. Found: C, 64.75; H, 6.57; N, 16.09; S, 12.26.

(E)-2-(2-(1-Octylpyridin-2(1H)-ylidene)ethylidene)malononitrile $(6 \mathrm{~g})$. Yield $86 \%, \mathrm{mp} 82-83{ }^{\circ} \mathrm{C}$, orange crystals. IR (KBr), v, cm ${ }^{-1}$ : 2957, 2919, 2855, 2188, 2175, 1634, 1569, 1528, 1497, 1478, 1440, 1406, 1322, $1305,1275,1228,1161,1076,1043,991,955,786,757,716,615,592,576 .{ }^{1} \mathrm{H}$ NMR, $\delta$, ppm: 0.83-0.85 (m, $\left.3 \mathrm{H},\left(8^{\prime}\right) \mathrm{CH}_{3}\right), 1.25-1.31\left(\mathrm{~m}, 10 \mathrm{H}, 5 \mathrm{CH}_{2}\right), 1.71\left(\mathrm{sbr}, 2 \mathrm{H},\left(2^{\prime}\right) \mathrm{CH}_{2}\right), 4.13\left(\mathrm{t}, 2 \mathrm{H},\left(1^{\prime}\right) \mathrm{CH}_{2}, J 7.3 \mathrm{~Hz}\right), 5.56(\mathrm{~d}, 1 \mathrm{H}$, (2) $\mathrm{CH}, 3$ J $13.8 \mathrm{~Hz}), 6.87$ (t, 1H, $\left.\mathrm{CH}_{p y}, J 6.6 \mathrm{~Hz}\right), 7.63\left(\mathrm{t}, 1 \mathrm{H}, \mathrm{CH}_{\mathrm{py}}, J 7.3 \mathrm{~Hz}\right), 7.88-7.95\left(\mathrm{~m}, 2 \mathrm{H}, \mathrm{CH}_{\mathrm{py}},(1) \mathrm{CH}\right), 8.07$ (d, $1 \mathrm{H}, \mathrm{CH}_{\mathrm{py}}, \mathrm{J} 6.6 \mathrm{~Hz}$ ). ${ }^{13} \mathrm{C} \mathrm{NMR}, \delta$, ppm: 14.37, 22.48, 26.14, 27.79, 28.76, 28.97, 31.51, 49.22, 55.48, 93.56, $115.42,118.89,121.05,121.21,138.55,142.03,149.53,152.72$. Anal. calcd for $\mathrm{C}_{18} \mathrm{H}_{23} \mathrm{~N}_{3}(281.40): \mathrm{C}, 76.83$; $\mathrm{H}, 8.24 ; \mathrm{N}, 14.93$. Found: $\mathrm{C}, 76.71 ; \mathrm{H}, 8.13 ; \mathrm{N}, 14.75$.

Ethyl (2E,4E)-2-cyano-4-(1-octylpyridin-2(1H)-ylidene)but-2-enoate (6h). Yield 84\%, mp 51-52 ${ }^{\circ} \mathrm{C}$, orange crystals. IR (KBr), v, cm ${ }^{-1}$ : 2954, 2928, 2856, 2188, 1674, 1633, 1565, 1531, 1446, 1419, 1311, 1221, 1155, $1095,1048,955,883,801,717 .{ }^{1} \mathrm{H} N M R, \delta, p p m: 0.81-0.84\left(\mathrm{sbr}, 3 \mathrm{H},\left(8^{\prime}\right) \mathrm{CH}_{3}\right), 1.17-1.31\left(\mathrm{~m}, 13 \mathrm{H}, \mathrm{CO}_{2} \mathrm{CH}_{2} \mathrm{C}_{3}\right.$, $\left.5 \mathrm{CH}_{2}\right), 1.71\left(\mathrm{sbr}, 2 \mathrm{H},\left(2^{\prime}\right) \mathrm{CH}_{2}\right), 4.07-4.10\left(\mathrm{~m}, 4 \mathrm{H},\left(1^{\prime}\right) \mathrm{CH}_{2}, \mathrm{CO}_{2} \mathrm{CH}_{2} \mathrm{CH}_{3}\right), 5.53(\mathrm{~d}, 1 \mathrm{H},(4) \mathrm{CH}, 3 \mathrm{~J} 14.0 \mathrm{~Hz}), 6.74(\mathrm{t}$, $\left.1 \mathrm{H}, \mathrm{CH}_{\mathrm{py}}, J 6.5 \mathrm{~Hz}\right), 7.55$ (t, $\left.1 \mathrm{H}, \mathrm{CH}_{\mathrm{py}}, J 7.7 \mathrm{~Hz}\right), 7.74\left(\mathrm{~d}, 1 \mathrm{H}, \mathrm{CH}_{\mathrm{py}}, J 8.9 \mathrm{~Hz}\right), 7.99-8.10\left(\mathrm{~m}, 2 \mathrm{H}, \mathrm{CH}_{\mathrm{py}},(3) \mathrm{CH}\right) .{ }^{13} \mathrm{C}$ NMR, $\delta$, ppm: 14.34, 15.08, 22.49, 26.19, 27.66, 28.82, 29.01, 31.54, 55.25, 58.83, 59.37, 75.83, 92.65, $114.44,120.33,120.74,138.08,141.92,147.39,153.10,166.32$. Anal. calcd for $\mathrm{C}_{20} \mathrm{H}_{28} \mathrm{~N}_{2} \mathrm{O}_{2}$ (328.46): C, 73.14; H, 8.59; N, 8.53. Found: C, 72.98; H, 8.42; N, 8.46.

(2E,4E)-4-(1-Octylpyridin-2(1H)-ylidene)-2-cyanobut-2-enethioamide (6i). Yield 88\%, mp $139-141{ }^{\circ} \mathrm{C}$, red

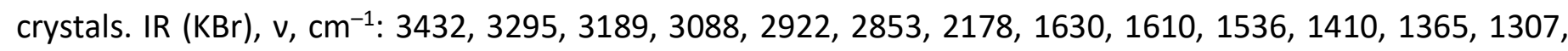
$1236,1217,1148,1056,1036,959,862,821,757,716,573,545 .{ }^{1} \mathrm{H} N M R, \delta, p p m: 0.85\left(\mathrm{t}, 3 \mathrm{H},\left(8^{\prime}\right) \mathrm{CH}_{3}, J 6\right.$ $\mathrm{Hz}), 1.25-1.32\left(\mathrm{~m}, 10 \mathrm{H}, 5 \mathrm{CH}_{2}\right), 1.73\left(\mathrm{sbr}, 2 \mathrm{H},\left(2^{\prime}\right) \mathrm{CH}_{2}\right), 4.11\left(\mathrm{t}, 2 \mathrm{H},\left(1^{\prime}\right) \mathrm{CH}_{2}, J 7.3 \mathrm{~Hz}\right), 5.57\left(\mathrm{~d}, 1 \mathrm{H},(4) \mathrm{CH}, 3^{3} \mathrm{j} .7\right.$ $\mathrm{Hz}), 6.82\left(\mathrm{t}, 1 \mathrm{H}, \mathrm{CH}_{p y}, J 6.7 \mathrm{~Hz}\right), 7.63\left(\mathrm{~d}, 1 \mathrm{H}, \mathrm{CH}_{p y}, J 7.3 \mathrm{~Hz}\right), 7.82\left(\mathrm{~d}, 1 \mathrm{H}, \mathrm{CH}_{p y}, J 9 \mathrm{~Hz}\right), 7.98-8.05\left(\mathrm{~m}, 3 \mathrm{H}, \mathrm{NH}_{2}\right.$, $\left.\mathrm{CH}_{\mathrm{py}}\right), 8.39$ (d, $\left.1 \mathrm{H},(3) \mathrm{CH},{ }^{3} \mathrm{~J} 13.7 \mathrm{~Hz}\right) .{ }^{13} \mathrm{C} \mathrm{NMR}, \delta, \mathrm{ppm}: 14.39,22.48,26.21,27.70,28.82,29.00,31.54$, $55.43,89.38,94.16,114.80,120.48,121.05,138.18,142.24,144.86,153.27,190.78$. Anal. calcd for $\mathrm{C}_{18} \mathrm{H}_{25} \mathrm{~N}_{3} \mathrm{~S}$ (315.48): C, 68.53; H, 7.99; N, 13.32; S, 10.16. Found: C, 68.48; H, 7.88; N, 13.13; S, 10.09.

tert-Butyl (E)-2-(2-(3,3-dicyanoallylidene)pyridin-1(2H)-yl)acetate (6j). Yield 65\%, mp 187-189 ${ }^{\circ} \mathrm{C}$, yellow crystals. IR (KBr), v, cm ${ }^{-1}$ : 2182, 1672, 1664, 1523, 1459, 1443, 1411, 1306, 1241, 1208, 1148, 1085, 1035,

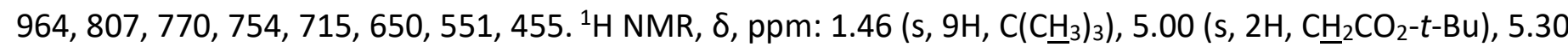
(d, $1 \mathrm{H},(2) \mathrm{CH}, 3 \mathrm{~J} 13.6 \mathrm{~Hz}), 6.86\left(\mathrm{t}, 1 \mathrm{H}, \mathrm{CH}_{\mathrm{py}}, J 6.7 \mathrm{~Hz}\right), 7.62\left(\mathrm{t}, 1 \mathrm{H}, \mathrm{CH}_{\mathrm{py}}, J 7.7 \mathrm{~Hz}\right) 7.92-7.97(\mathrm{~m}, 3 \mathrm{H},(1) \mathrm{CH}$, $\left.2 \mathrm{CH}_{\text {py }}\right) .{ }^{13} \mathrm{C} N M R, \delta$, ppm: 27.98, 50.96, 57.20, 83.84, 93.45, 114.84, 118.40, 120.59, 121.01, 138.80, 142.44, 150.28, 153.57, 166.09. Anal. calcd for $\mathrm{C}_{16} \mathrm{H}_{17} \mathrm{~N}_{3} \mathrm{O}_{2}$ (283.33): C, 67.83; $\mathrm{H}, 6.05 ; \mathrm{N}, 14.83$. Found: $\mathrm{C}, 67.71 ; \mathrm{H}$, $5.94 ; \mathrm{N}, 14.70$.

Ethyl (2E,4E)-4-(1-(2-(tert-butoxy)-2-oxoethyl)pyridin-2(1H)-ylidene)-2-cyanobut-2-enoate (6k). Yield 63\%, $\mathrm{mp} 171-173{ }^{\circ} \mathrm{C}$, orange crystals. IR (KBr), v, cm ${ }^{-1}$ : 3090, 3055, 2986, 2974, 2904, 2187, 1731, 1669, 1637, 1525, 1458, 1417, 1370, 1310, 1231, 1152, 1091, 1046 1023, 967, 952, 877, 801, 772, 756, 720. ${ }^{1} \mathrm{H}$ NMR, $\delta$, ppm: $1.21\left(\mathrm{t}, 2 \mathrm{H}, \mathrm{CO}_{2} \mathrm{CH}_{2} \underline{\mathrm{C}}_{3}, J 7 \mathrm{~Hz}\right)$ 1.44-1.47 (m, 9H, C( $\left.\left.\underline{\mathrm{C}}_{3}\right)_{3}\right), 4.10\left(\mathrm{q}, 2 \mathrm{H}, \mathrm{CO}_{2} \mathrm{C}_{2} \mathrm{CH}_{3}, J 7 \mathrm{~Hz}\right), 4.94(\mathrm{~s}, 2 \mathrm{H}$, $\left.\mathrm{C}_{2} \mathrm{CO}_{2} t-\mathrm{Bu}\right), 5.30\left(\mathrm{~d}, 1 \mathrm{H},(4) \mathrm{CH},{ }^{3} \mathrm{~J} 13.7 \mathrm{~Hz}\right), 6.76\left(\mathrm{t}, 1 \mathrm{H}, \mathrm{CH}_{\mathrm{py}}, J 7.7 \mathrm{~Hz}\right), 7.56\left(\mathrm{t}, 1 \mathrm{H}, \mathrm{CH}_{\mathrm{py}}, J 7.7 \mathrm{~Hz}\right), 7.74(\mathrm{~d}$, $\left.1 \mathrm{H}, \mathrm{CH}_{\mathrm{py}}, J 9 \mathrm{~Hz}\right), 7.90\left(\mathrm{~d}, 1 \mathrm{H}, \mathrm{CH}_{\mathrm{py}}, J 6.5 \mathrm{~Hz}\right), 8.09\left(\mathrm{~d},(3) \mathrm{CH}, 1 \mathrm{H},{ }^{3} \mathrm{~J} 13.7 \mathrm{~Hz}\right) .{ }^{13} \mathrm{C} \mathrm{NMR}, \delta, \mathrm{ppm}: 15.03,27.99$, 
57.01, 59.62, 77.48, 83.74, 92.51, 113.80, 119.87, 120.57, 138.25, 142.35, 147.86, 153.87, 166.11, 166.16. Anal. calcd for $\mathrm{C}_{18} \mathrm{H}_{22} \mathrm{~N}_{2} \mathrm{O}_{4}$ (330.38): $\mathrm{C}, 65.44 ; \mathrm{H}, 6.71 ; \mathrm{N}, 8.48$. Found: $\mathrm{C}, 65.27 ; \mathrm{H}, 6.61 ; \mathrm{N}, 8.34$.

tert-Butyl 2-((E)-2-((E)-4-amino-3-cyano-4-thioxobut-2-en-1-ylidene)pyridin-1(2H)-yl)acetate (6I). Yield

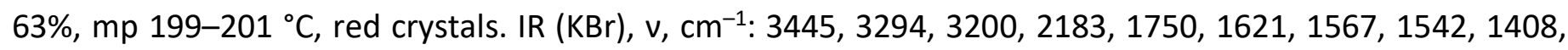
$1368,1303,1282,1242,1226,1162,1145,1048,864,852,803,768,747,697,667,558,537 .{ }^{1} \mathrm{H} N M R, \delta$, ppm: 1.47 (s, 9H, C(C $\left.\left.\underline{\mathrm{H}}_{3}\right)_{3}\right), 4.94\left(\mathrm{~s}, 2 \mathrm{H}, \mathrm{CH}_{2} \mathrm{CO}_{2}-t-\mathrm{Bu}\right), 5.30\left(\mathrm{~d}, 1 \mathrm{H},(4) \mathrm{CH},{ }^{3} \mathrm{~J} 13.3 \mathrm{~Hz}\right), 6.78\left(\mathrm{t}, 1 \mathrm{H}, \mathrm{CH}_{\mathrm{py}}, J 6.4\right.$ $\mathrm{Hz}), 7.61\left(\mathrm{t}, 1 \mathrm{H}, \mathrm{CH}_{\mathrm{py}}, J 7.4 \mathrm{~Hz}\right), 7.80\left(\mathrm{~d}, 1 \mathrm{H}, \mathrm{CH}_{\mathrm{py}}, J 8.9 \mathrm{~Hz}\right), 7.91\left(\mathrm{~d}, 1 \mathrm{H}, \mathrm{CH}_{\mathrm{py}}, J 6.4 \mathrm{~Hz}\right), 8.10\left(\mathrm{sbr}, 2 \mathrm{H}, \mathrm{NH}_{2}\right)$, 8.35 (d, (3) CH, $\left.1 \mathrm{H},{ }^{3} \mathrm{~J} 13.3 \mathrm{~Hz}\right) .{ }^{13} \mathrm{C}$ NMR, $\delta$, ppm: 28.01, 57.03, 83.78, 91.05, 93.64, 113.92, 120.01, 120.79, 138.14, 142.56, 144.86, 153.98. Anal. calcd for $\mathrm{C}_{16} \mathrm{H}_{19} \mathrm{~N}_{3} \mathrm{O}_{2} \mathrm{~S}$ (317.41): C, 60.55; H, 6.03; N, 13.24; S, 10.10. Found: C, 60.48; H, 5.92; N, 13.11; S, 10.06 .

2-(2-(1-Methylpyridin-4(1H)-ylidene)ethylidene)malononitrile (7a). Yield $68 \%, \mathrm{mp}>260{ }^{\circ} \mathrm{C}$, orange crystals. IR (KBr), v, cm $\mathrm{cm}^{-1}$ : 2189, 2164, 1649, 1560, 1538, 1517, 1485, 1310, 1278, 1181, 1041, 954, 847, 804, 708,

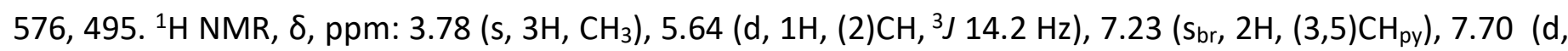
$\left.1 \mathrm{H},(1) \mathrm{CH},{ }^{3} \mathrm{~J} 14.2 \mathrm{~Hz}\right), 7.89$ (d, 2H, (2,6) $\left.\mathrm{CH}_{\mathrm{py}}, J 6.7 \mathrm{~Hz}\right) .{ }^{13} \mathrm{C} \mathrm{NMR}, \delta, \mathrm{ppm}: 44.56,47.73,102.54,119.48$, 121.66, 141.66, 147.88, 152.84. Anal. calcd for $\mathrm{C}_{11} \mathrm{H}_{9} \mathrm{~N}_{3}$ (183.21): C, 72.11; H, 4.95; N, 22.94. Found: C, 72.06; H, 4.92; N, 22.65.

Ethyl (Z)-2-cyano-4-(1-methylpyridin-4(1H)-ylidene)but-2-enoate (7b). Yield 68\%, mp 211-213 ${ }^{\circ} \mathrm{C}$, orange crystals. IR (KBr), v, cm${ }^{-1}$ : 2192, 1668, 1576, 1516, 1492, 1460, 1416, 1364, 1308, 1276, 1256, 1228, 1208, 1168, 1100, 1016, 948, 844, 812, 756, 668, 484. ${ }^{1} \mathrm{H} \mathrm{NMR}, \delta, \mathrm{ppm}: 1.18$ (t, 3H, $\left.\mathrm{CO}_{2} \mathrm{CH}_{2} \mathrm{CH}_{3}, J 7 \mathrm{~Hz}\right), 3.77$ (s, 3H, $\left.\mathrm{CH}_{3}\right), 4.06$ (q, $\left.2 \mathrm{H}, \mathrm{CO}_{2} \mathrm{CH}_{2} \mathrm{CH}_{3}, J \mathrm{~Hz}\right), 5.62(\mathrm{~d}, 1 \mathrm{H},(4) \mathrm{CH}, 3 \mathrm{~J} 14.3 \mathrm{~Hz}), 7.14\left(\mathrm{sbr}, 2 \mathrm{H},(3,5) \mathrm{CH}_{\mathrm{py}}\right), 7.79(\mathrm{~d}, 2 \mathrm{H}$, $\left.(2,6) \mathrm{CH}_{\mathrm{py}}, \mathrm{J} 6.8 \mathrm{~Hz}\right), 7.95$ (d, $\left.1 \mathrm{H},(3) \mathrm{CH},{ }^{3} \mathrm{~J} 14.3 \mathrm{~Hz}\right) .{ }^{13} \mathrm{C} \mathrm{NMR}, \delta, \mathrm{ppm}: 15.11,44.36,59.33,74.27,74.50$, $101.87,120.93,141.27,146.00,153.09,166.75$. Anal. calcd for $\mathrm{C}_{13} \mathrm{H}_{14} \mathrm{~N}_{2} \mathrm{O}_{2}$ (230.27): C, 67.81; $\mathrm{H}, 6.13 ; \mathrm{N}$, 12.17. Found: C, 67.78; $\mathrm{H}, 5.88 ; \mathrm{N}, 12.12$.

(Z)-2-Cyano-4-(1-methylpyridin-4(1H)-ylidene)but-2-enethioamide (7c). Yield 50\%, mp $216-218{ }^{\circ} \mathrm{C}$, vinous-colored crystals. IR (KBr), v, cm $\mathrm{cm}^{-1}$ : 3336, 3299, 3173, 2182, 1643, 1573, 1477, 1427, 1416, 1385, $1311,1251,1186,1121,1033,948,879,853,782,718,647,599,494 .{ }^{1} \mathrm{H} N M R, \delta, p p m: 3.79\left(\mathrm{~s}, 3 \mathrm{H}, \mathrm{CH}_{3}\right)$, $5.65\left(\mathrm{~d}, 1 \mathrm{H},(4) \mathrm{CH},{ }^{3} \mathrm{~J} 13.8 \mathrm{~Hz}\right), 7.20\left(\mathrm{sbr}, 2 \mathrm{H},(3,5) \mathrm{CH}_{\mathrm{py}}\right), 7.86\left(\mathrm{~m}, 4 \mathrm{H}, \mathrm{NH}_{2},(2,6) \mathrm{CH}_{\mathrm{py}}\right), 8.25(\mathrm{~d}, 1 \mathrm{H},(3) \mathrm{CH}, 3 \mathrm{~J}$ 13.8 Hz). ${ }^{13} \mathrm{C}$ NMR, $\delta$, ppm: 44.47, 88.19, 103.28, 116.97, 117.41, 120.82, 141.32, 143.80, 153.43, 190.58. Anal. calcd for $\mathrm{C}_{11} \mathrm{H}_{11} \mathrm{~N}_{3} \mathrm{~S}$ (217.29): C, 60.80; H, 5.10; N, 19.34; S, 14.75. Found: C, 60.77; H, 5.03; N, 19.17; S, 14.62.

2-(2-(1-Butylpyridin-4(1H)-ylidene)ethylidene)malononitrile (7d). Yield 67\%, mp 171-173 ${ }^{\circ} \mathrm{C}$, orange crystals. IR (KBr), v, cm${ }^{-1}$ : 3072, 2960, 2933, 2863, 2190, 2168, 1648, 1549, 1512, 1487, 1441, 1397, 1313,

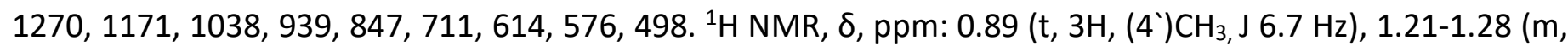
$\left.2 \mathrm{H},\left(3^{\prime}\right) \mathrm{CH}_{2}\right), 1.67-1.76\left(\mathrm{~m}, 2 \mathrm{H},\left(2^{\prime}\right) \mathrm{CH}_{2}\right), 4.03\left(\mathrm{t}, 2 \mathrm{H},\left(1^{\prime}\right) \mathrm{CH}_{2}, J 7 \mathrm{~Hz}\right), 5.66\left(\mathrm{~d}, 1 \mathrm{H},(2) \mathrm{CH}, 3^{3} J 14.1 \mathrm{~Hz}\right), 7.21(\mathrm{sbr}$, $\left.2 \mathrm{H},(3,5) \mathrm{CH}_{p y}\right) 7.74\left(\mathrm{~d},(1) \mathrm{CH}, 1 \mathrm{H},{ }^{3} J 14.1 \mathrm{~Hz}\right), 7.98\left(\mathrm{~d}, 2 \mathrm{H},(2,6) \mathrm{CH}_{\mathrm{py}}, J 6.9 \mathrm{~Hz}\right) .{ }^{13} \mathrm{C} \mathrm{NMR}, \delta, \mathrm{ppm} .:$ 13.81, 19.25, $32.69,48.11,57.05,102.61,119.41,121.60,128.81,140.77,148.09,153.00,155.00$. Anal. calcd for $\mathrm{C}_{14} \mathrm{H}_{15} \mathrm{~N}_{3}$ (225.30): C, 74.64; H, 6.71; N, 18.65. Found: C, 74.55; H, 6.60; N, 18.52.

Ethyl (Z)-2-cyano-4-(1-butylpyridin-4(1H)-ylidene)but-2-enoate (7e). Yield 68\%, mp 139-141 ${ }^{\circ} \mathrm{C}$, orange crystals. IR (KBr), v, cm $\mathrm{cm}^{-1}$ : 3060, 2964, 2936, 2896, 2192, 1656, 1527, 1492, 1420, 1332, 1256, 1224, 1192, $1104,948,848,820,756,540,504 .{ }^{1} \mathrm{H} N M R, \delta, p p m: 0.89-0.92\left(\mathrm{~m}, 3 \mathrm{H},\left(4^{\prime}\right) \mathrm{CH}_{3}\right), 1.17-1.24\left(\mathrm{~m}, 5 \mathrm{H},\left(3^{\prime}\right) \mathrm{CH}_{2}\right.$, $\left.\mathrm{CO}_{2} \mathrm{CH}_{2} \mathrm{CH}_{3}\right), 1.70\left(\mathrm{sbr}, 2 \mathrm{H},\left(2^{\prime}\right) \mathrm{CH}_{2}\right), 4.01-4.06\left(\mathrm{~m}, 4 \mathrm{H},\left(1^{\prime}\right) \mathrm{CH}_{2}, \mathrm{CO}_{2} \mathrm{CH}_{2} \mathrm{CH}_{3}\right), 5.63\left(\mathrm{~d}, 1 \mathrm{H},(4) \mathrm{CH}, 3^{3} \mathrm{j} 14.3 \mathrm{~Hz}\right)$, $7.25\left(\mathrm{~s}_{\mathrm{br}}, 2 \mathrm{H},(3,5) \mathrm{CH}_{\mathrm{py}}\right), 7.87-7.99\left(\mathrm{~m}, 3 \mathrm{H},(2,6) \mathrm{CH}_{\mathrm{py}},(3) \mathrm{CH}\right) .{ }^{13} \mathrm{C} \mathrm{NMR}, \delta, \mathrm{ppm}: 13.83,15.12,19.26,32.71$, 56.78, 59.30, 101.89, 120.77, 140.93, 140.48, 146.24, 153.20, 166.61. Anal. calcd for $\mathrm{C}_{16} \mathrm{H}_{20} \mathrm{~N}_{2} \mathrm{O}_{2}$ (272.35): C, 
70.56; H, 7.40; N, 10.29. Found: C, 70.49; H, 7.25; N, 10.10 .

(Z)-2-Cyano-4-(1-butylpyridin-4(1H)-ylidene)but-2-enethioamide (7f). Yield 62\%, $\mathrm{mp} 182-184^{\circ} \mathrm{C}$, vinouscolored crystals. IR (KBr), v, cm ${ }^{-1}$ : 3405, 3274, 3174, 2958, 2927, 2857, 2171, 1641, 1613, 1556, 1544, 1475, $1411,1369,1315,1255,1171,1035,870,860,764,640,559,506 .{ }^{1} \mathrm{H} N M R, \delta, p p m: 0.89\left(\mathrm{t}, 3 \mathrm{H},\left(4^{\prime}\right) \mathrm{CH}_{3}, \mathrm{~J} 6.9\right.$ $\mathrm{Hz}), 1.22-1.29\left(\mathrm{~m}, 2 \mathrm{H},\left(3^{\prime}\right) \mathrm{CH}_{2}\right), 1.69-1.74\left(\mathrm{~m}, 2 \mathrm{H},\left(2^{\prime}\right) \mathrm{CH}_{2}\right), 4.01\left(\mathrm{t}, 2 \mathrm{H},\left(1^{\prime}\right) \mathrm{CH}_{2}, \mathrm{~J} 6.9 \mathrm{~Hz}\right), 5.65\left(\mathrm{~d}, 1 \mathrm{H},(4) \mathrm{CH}, 3^{3} \mathrm{~J}\right.$ $13.2 \mathrm{~Hz}), 7.18\left(\mathrm{sbr}, 2 \mathrm{H},(3,5) \mathrm{CH}_{\mathrm{py}}\right), 7.9-7.94\left(\mathrm{~m}, 4 \mathrm{H},(2,6) \mathrm{CH}_{\mathrm{py}}, \mathrm{NH}_{2}\right), 8.25(\mathrm{~d}, 1 \mathrm{H},(3) \mathrm{CH}, 3 \mathrm{~J} 13.2 \mathrm{~Hz}) .{ }^{13} \mathrm{C} \mathrm{NMR}$, $\delta$, ppm: 13.84, 19.29, 32.70, 56.96, 88.59, 103.29, 120.78, 140.43, 143.74, 153.56, 190.61. Anal. calcd for $\mathrm{C}_{14} \mathrm{H}_{17} \mathrm{~N}_{3} \mathrm{~S}$ (259.37): C, 64.83; H, 6.61; N, 16.20; S, 12.36. Found: C, 64.77; H, 6.51; N, 16.14; S, 12.21.

2-(2-(1-Octylpyridin-4(1H)-ylidene)ethylidene)malononitrile $\mathbf{( 7 g )}$. Yield $67 \%, \mathrm{mp} 137-139^{\circ} \mathrm{C}$, orange crystals. IR (KBr), v, cm ${ }^{-1}$ : 3071, 2945, 2624, 2855, 2193, 2173, 1648, 1539, 1515, 1487, 1456, 1434, 1399, $1376,1329,1311,1267,1212,1161,1036,958,856,807,723,586,576,513 .{ }^{1} \mathrm{H}$ NMR, $\delta, p p m: 0.85$ (t, $3 \mathrm{H}$, $\left.\left(8^{\prime}\right) \mathrm{CH}_{3}\right), 1.24\left(\mathrm{sbr}, 10 \mathrm{H}, 5 \mathrm{CH}_{2}\right), 1.73\left(\mathrm{~s}_{\mathrm{br}}, 2 \mathrm{H},\left(2^{\prime}\right) \mathrm{CH}_{2}\right), 4.02\left(\mathrm{t}, 2 \mathrm{H},\left(1^{\prime}\right) \mathrm{CH}_{2}, J 7 \mathrm{~Hz}\right), 5.68(\mathrm{~d}, 1 \mathrm{H},(2) \mathrm{CH}, 3 \mathrm{~J} 13.2$ $\mathrm{Hz}), 7.27\left(\mathrm{sbr}, 2 \mathrm{H},(3,5) \mathrm{CH}_{\mathrm{py}}\right), 7.75\left(\mathrm{~d}, 1 \mathrm{H},(1) \mathrm{CH},{ }^{3} \mathrm{~J} 13.2 \mathrm{~Hz}\right), 7.97\left(\mathrm{~d}, 2 \mathrm{H},(2,6) \mathrm{CH}_{\mathrm{py}}, J 6.9 \mathrm{~Hz}\right) .{ }^{13} \mathrm{C} \mathrm{NMR}, \delta$, ppm.: 14.37, 22.49, 25.94, 28.84, 28.95, 30.67, 31.59, 48.19, 57.29, 102.60, 119.38, 121.56, 140.75, 148.12, 152.99. Anal. calcd for $\mathrm{C}_{18} \mathrm{H}_{23} \mathrm{~N}_{3}$ (281.40): C, 76.83; $\mathrm{H}, 8.24 ; \mathrm{N}, 14.93$. Found: $\mathrm{C}, 76.75 ; \mathrm{H}, 8.07 ; \mathrm{N}, 14.81$.

Ethyl (Z)-2-cyano-4-(1-octylpyridin-4(1H)-ylidene)but-2-enoate (7h). Yield 71\%, $\mathrm{mp} 131-133{ }^{\circ} \mathrm{C}$, red crystals. IR (KBr), v, cm ${ }^{-1}$ : 3063, 2953, 2924, 2855, 2192, 1656, 1574, 1517, 1490, 1422, 1258, 1225, 1194, 1106, 1029, 946, 847, 822, 756. ${ }^{1} \mathrm{H}$ NMR, $\delta, \mathrm{ppm}: 0.84$ (t, 3H, $\left.\left(8^{\prime}\right) \mathrm{CH}_{3}\right), 1.17-1.22\left(\mathrm{~m}, 13 \mathrm{H}, 5 \mathrm{CH}_{2}, \mathrm{CO}_{2} \mathrm{CH}_{2} \mathrm{C}_{3}\right)$, 1.71-1.73 (m, 2H, (2 $\left.) \mathrm{CH}_{2}\right)$, 3.96-4.11 (m, 4H, (1 $\left.\left.{ }^{\prime}\right) \mathrm{CH}_{2}, \mathrm{CO}_{2} \mathrm{C}_{2} \mathrm{CH}_{3}\right), 5.64\left(\mathrm{~d}, 1 \mathrm{H},(4) \mathrm{CH},{ }^{3} \mathrm{~J} 14.3 \mathrm{~Hz}\right), 7.09$ (sbr, $\left.2 \mathrm{H},(3,5) \mathrm{CH}_{\mathrm{py}}\right), 7.85\left(\mathrm{~d}, 2 \mathrm{H},(2,6) \mathrm{CH}_{\mathrm{py}}, J \mathrm{~Hz}\right), 7.97\left(\mathrm{~d}, 1 \mathrm{H},(3) \mathrm{CH},{ }^{3} \mathrm{~J} 14.3 \mathrm{~Hz}\right) .{ }^{13} \mathrm{C} \mathrm{NMR}, \delta, \mathrm{ppm}: 14.35,15.10$, 22.49, 25.97, 28.87, 28.97, 30.70, 31.60, 57.03, 59.29, 75.04, 101.89, 120.71, 140.27, 146.26, 153.14, 166.58. Anal. calcd for $\mathrm{C}_{20} \mathrm{H}_{28} \mathrm{~N}_{2} \mathrm{O}_{2}$ (328.46): C, 73.14; $\mathrm{H}, 8.59 ; \mathrm{N}, 8.53$. Found: $\mathrm{C}, 73.01 ; \mathrm{H}, 8.48 ; \mathrm{N}, 8.40$.

(Z)-2-Cyano-4-(1-octylpyridin-4(1H)-ylidene)but-2-enethioamide (7i). Yield 85\%, mp 142-143 ${ }^{\circ} \mathrm{C}$, red crystals. IR (KBr), v, cm cm $^{-1}$ 3356, 3274, 3152, 2924, 2853, 2173, 1648, 1633, 1548, 1479, 1413, 1359, 1316, 1233, 1201, 1163, 1031, 962, 798, 758, 656. ${ }^{1} \mathrm{H}$ NMR, $\delta, p p m: 0.85$ (t, 3H, $\left.\left(8^{\circ}\right) \mathrm{CH}_{3}, J 5.9 \mathrm{~Hz}\right), 1.24(\mathrm{~s}, 10 \mathrm{H}$, $\left.5 \mathrm{CH}_{2}\right), 1.73\left(\mathrm{sbr}_{\mathrm{b}}, 2 \mathrm{H},\left(2^{\prime}\right) \mathrm{CH}_{2}\right), 4.00\left(\mathrm{t}, 2 \mathrm{H},\left(1^{\prime}\right) \mathrm{CH}_{2}, J 7.1 \mathrm{~Hz}\right), 5.66(\mathrm{~d}, 1 \mathrm{H},(4) \mathrm{CH}, 3 \mathrm{~J} 14.0 \mathrm{~Hz}), 7.18\left(\mathrm{~s}_{\mathrm{br}}, 2 \mathrm{H}\right.$, $\left.(3,5) \mathrm{CH}_{\mathrm{py}}\right), 7.88-7.94\left(\mathrm{~m}, 4 \mathrm{H},(2,6) \mathrm{CH}_{\mathrm{py}}, \mathrm{NH}_{2}\right), 8.25\left(\mathrm{~d}, 1 \mathrm{H},(3) \mathrm{CH},{ }^{3} \mathrm{~J} 14.0 \mathrm{~Hz}\right) .{ }^{13} \mathrm{C} \mathrm{NMR}, \delta, \mathrm{ppm}: 14.38,22.49$, 25.98, 28.97, 30.68, 31.59, 57.17, 88.68, 103.26, 118.40, 120.75, 125.06, 129.95, 140.41, 143.76, 153.54, 190.66. Anal. calcd for $\mathrm{C}_{18} \mathrm{H}_{25} \mathrm{~N}_{3} \mathrm{~S}$ (315.48): C, 68.53; $\mathrm{H}, 7.99 ; \mathrm{N}, 13.32 ; \mathrm{S}, 10.16$. Found: $\mathrm{C}, 68.51 ; \mathrm{H}, 7.85 ; \mathrm{N}$, 13.19; S, 10.04 .

\section{References}

1. Shirinian, V.Z.; Shimkin, A. A. Heterocyclic Polymethine Dyes; Strekowski, L. Eds.; Springer: Verlag

2. Kulinich, A. V.; Ischenko, A. A. Russ. Chem. Rev. 2009, 78, 2, 141-164.

https://doi.org/10.1070/RC2009v078n02ABEH003900

3. Ischenko, A. A. Usp. Khim. 1991, 60, 1708-1743.

4. Klunich, A. V.; Ischenko, A.A.; Shishkina, S. V.; Konovalova, I. S.; Shishkin, O. V. Zh. Strukt. Khim. 2007, 48, 5, 971-978.

5. Brooker, L. G. S.; Craig, A. C.; Heseltine, D. W.; Jenkins, P. W.; Lincoln, L. L. J. Am. Chem. Soc. 1965, 87, 11, 2443.

6. Klunich, A. V.; Derevyanko, N. A.; Ischenko, A. A. Zh. Obshch. Khim. 2006, 76, 9, 1441.

7. Kakehi, A.; Ito, S.; Matsubara, K. Bull. Chem. Soc. Jpn. 1995, 68, 8, 2409-2415. 
https://doi.org/10.1246/bcsj.68.2409

8. Kakehi, A.; Ito, S.; Ohizumi, T.; Maeda, T. J. Org. Chem. 1982, 47, 2, 369-371.

https://doi.org/10.1021/jo00341a042

9. Kakehi, A.; Ito, S.; Maeda, T.; Takeda, T.; Nishimura, N.; Tamashima, M.; Yamaguehi, T. J. Org. Chem. 1978, 43, 25, 4837-4840.

https://doi.org/10.1021/jo00419a026

10. Strell, V. M.; Braunbruck, W. B.; Reithmayr, L. Justus Liebigs Ann. Chem. 1954, 587.

11. Kakehi, A.; Ito, S.; Funahashi, T.; Ogasawara, N. Bull. Chem. Soc. Jpn. 1976, 49, 8, 2250-2252. https://doi.org/10.1246/bcsj.49.2250

12. Bespalov, B. P.; Abolin, A. G.; Rumyantsev, V. G. Khim. Geterotsikl. Soedin. 1985, 5, 603-608.

13. Becher, J.; Hansen, P. J. Heterocyclic Chem. 1988, 25, 367-371.

https://doi.org/10.1002/jhet.5570250203

14. Peng, Z.-H.; Qun, L.; Zbou, X.-F.; Carroll, S.; Geise, H. J.; Peng, B.-X.; Dommisse, R.; Carleer, R. J. Mater. Chem. 1996, 6, 4, 559-565.

https://doi.org/10.1039/jm9960600559

15. Klunich, A. V.; Derevyanko, N. A.; Ischenko, A. A. Izv. Akad. Nauk, Ser. Khim. 2005, 12, 2726.

16. Mishra, A.; Behera, R.K.; Behera, P.K.; Mishra, B.K.; Behera, G.B. Cyanines during the 1990s: A Review; Chem. Rev. 2000, (100), 6, pp.1973-2011.

https://doi.org/10.1021/cr990402t

17. Carreon, J. R.; Stewart, K. M.; Mahon Jr., K. P.; Shin, S.; Kelley, S. O. Bioorg. Med. Chem. Lett. 2007, 17, 5182-5185.

https://doi.org/10.1016/j.bmcl.2007.06.097

18. Khoroshilov, G.; Demchak, I.; Saraeva, T. Synthesis, 2008, 10, 1541-1544.

https://doi.org/10.1055/s-2008-1072578

19. Tverdokhleb, N. M.; Khoroshilov, G. E.; Dotsenko, V. V. Tetrahedron Lett. 2014, 55, 48, 6593-6595. https://doi.org/10.1016/j.tetlet.2014.10.046

20. VanAllan, J. A.; Reynolds, G. A. J. Heterocycl. Chem. 1971, 8, 367-371.

https://doi.org/10.1002/jhet.5570080303

21. Wurthner, F.; Yao, S.; Debaerdemaeker, T.; Wortmann, R. J. Am. Chem. Soc. 2002, 124, 32, 9431-9447. https://doi.org/10.1021/ja020168f

22. Fortuna, C. G.; Barresi, V.; Bonaccorso, C.; Consiglio, G.; Failla, S.; Trovat-Salinao, A.; Musumarra, G. Eur. J. Med. Chem. 2012, 47, 221-227.

23. Nicholl, L.; Tarsio, P. J.; Blohm, H. U.S. Patent 2824 121, 1958, Chem. Abstr. 1958, 52, 11909.

24. Li, C.; Shaoqing, C.; Christophe, M. U.S. Patent 20064046 A1, 2006.

25. Dotsenko, V. V.; Krivokolysko, S. G.; Shishkina, S. V.; Shishkin, O. V. Russ. Chem. Bul. 2012, 61, 11, 2082-2087.

https://doi.org/10.1007/s11172-012-0291-3

26. Malakooti, R.; Mahmoudi, H.; Hosseinbadi, R.; Petrov, S.; Migliori, A. RSC Adv. 2013, 3, 22353. https://doi.org/10.1039/c3ra44682d

27. Ding, L.; Li, H.; Zhang, Y.; Zhang, K.; Yuan, H.; Wu, Q.; Jiao, Q.; Shi, D. RSC Adv. 2015, 5, 21415. https://doi.org/10.1039/C5RA01700A

28. Khidre, M. D.; Yakout, E.-S. M. A.; Mahran, M. R. M. Phosphorus, Sulfur, Silicon Relat. Elem. 1998, 133, 119. 
https://doi.org/10.1080/10426509808032459

29. Acheson, R. M.; Woolard J. J. Chem. Soc., Perkin 1 1975, 744-748.

https://doi.org/10.1039/p19750000744

30. Bach, G.; Daehne, S. Cyanine dyes and related compounds; Sainsbury, M. Eds.; Elsevier: Amsterdam, 1997; Vol. IVB, pp. $383-481$.

31. Abdel-Halim, S. T., Awad, M. T. J.Mol. Struct. 2005, 754, 16-24.

https://doi.org/10.1016/i.molstruc.2005.06.010 